\title{
Percepção de estudantes cegos sobre características geométricas 3D utilizando sistemas hápticos
}

Patricia Blini Estivalete - PPGIE/UFRGS, pestivalete@gmail.com

Eliseo Berni Reategui - PPGIE/UFRGS, eliseoreategui@gmail.com

Resumo: As tecnologias hápticas de software e hardware permitem o toque em objetos virtuais de uma cena com três direções e com feedback de força para que os usuários percebam a sensibilidade háptica dos efeitos, das texturas, das formas, do peso e das dimensões. O objetivo desta pesquisa é verificar como os sistemas hápticos apoiam os estudantes cegos na percepção de propriedades geométricas $2 \mathrm{D}$ ou $3 \mathrm{D}$. A revisão sistemática foi guiada pelo processo de Kitchenham (2004). Dessa maneira, as buscas foram realizadas de setembro a outubro de 2017 em bases de dados internacionais Springer, Sage Journals, ACM Digital Library (Association for Computing Machinery) e IEEE Xplore Digital Library - com o propósito de identificar trabalhos na área da ciência da computação, interação humano-computador, seguindo o protocolo de investigação entre os anos de 2000 a 2017. Os resultados demonstram que os Ambientes Virtuais Hápticos permitem aos estudantes cegos a identificação das características das formas geométricas 3D apesar das limitações de exploração possibilitadas pelos dispositivos hápticos. Dessa forma, as tecnologias hápticas de hardware e software podem proporcionar aos estudantes cegos a compreensão dos conceitos geométricos por meio de vários cenários de problemas contextualizados com o mundo real ou com a própria ciência matemática.

Palavras-chave: percepção háptica; cegos; geometria; tecnologia háptica.

\section{Perception of blind students on 3D geometric features using haptic systems}

\begin{abstract}
The software and hardware haptic technologies enable the touch of virtual objects in a three-way scene and with feedback of strength so that users perceive the haptic sensitivity of effects, textures, shapes, weight and dimensions. The aim of this research is to verify how the haptic systems support blind students in the perception of 2D or 3D geometric properties. The systematic review was guided by the Kitchenham process (2004). In this way, the searches were carried out from September to October 2017 in international databases - Springer, Sage Journals, ACM Digital Library (Association for Computing Machinery) and IEEE Xplore Digital Library - for the purpose of identifying works in Area of computer science, human-machine interaction, following the protocol of investigation between the years of 2000 to 2017. The results demonstrate that the haptic virtual environments allow blind students to identify the characteristics of 3D geometric shapes despite the limitations of exploitation made possible by the haptic devices. Thus, hardware and software haptic technologies can provide blind students with understanding of geometric concepts through various scenarios of contextualized problems with the real world or mathematical science itself. Keywords: Haptic Perception; Blind; Geometry; Haptic Technology
\end{abstract}

\section{Introdução}

Pesquisadores da área de ensino de geometria para estudantes cegos evidenciam algumas barreiras que devem ser superadas, como a compreensão de conceitos por meio da fala do professor e a ausência de material didático específico. Essas barreiras levam os estudantes cegos a sentirem-se excluídos do processo de ensino e aprendizagem, pois sem uma sequência didática apropriada e sem recursos táteis que apoiem a construção 
de conhecimentos geométricos, seus conhecimentos baseiam-se somente em memorização.

A pesquisa na área tem evidenciado a necessidade de desenvolvimento de recursos que possibilitem aos estudantes cegos experiências de aprendizagem mais próximas daquelas vivenciadas por seus colegas videntes. Lirio (2006) aponta, por exemplo, que os estudantes cegos, muitas vezes, se isolam por falta de material de apoio adequado ou por não conseguirem acompanhar o conteúdo apresentado pelo professor. Os estudantes fazem queixas sobre a desconexão do que aprendem na escola com o que poderia servir para a vida, sobretudo pelas dificuldades de aprendizagem impostas pela inadequação dos materiais de apoio (Ferronato, 2002).

Lirio (2006) observa que a maioria dos professores não possui o conhecimento necessário para desenvolver recursos didáticos de apoio para os conteúdos de geometria, conduzindo os estudantes cegos a um ensino mais superficial e à abstração para compreensão dos assuntos trabalhados, o que produz uma falta de significado sobre aquilo que foi ensinado. Os estudantes, da mesma forma, evidenciaram problemas de aprendizagem em geometria em virtude da falta de capacitação dos professores em relação a métodos de ensino que facilitem a compreensão dos conceitos por meio da percepção tátil (Argyropoulos, 2002). Nesse sentido, Fernandes (2004) mostra a importância dos estímulos adequados por intermédio do tato, do áudio e da fala, para que os estudantes cegos tenham oportunidades de ensino e assim consigam construir conhecimentos relacionados aos conteúdos de matemática.

Devido aos resultados de pesquisas que mostram as dificuldades de aprendizagem dos estudantes cegos em função da falta de recursos didáticos que facilitem a compreensão dos conceitos de matemática por meio dos sentidos háptico e auditivo, estão surgindo novas oportunidades de pesquisa na área da Informática na Educação, com vistas a oportunizar o uso de tecnologias hápticas no ensino e na aprendizagem de geometria como forma de inclusão dos estudantes cegos.

Com base nessa compreensão, este trabalho tem como objetivo mapear, por meio de uma Revisão Sistemática de Literatura, como os estudantes cegos percebem as propriedades geométricas 3D usando sistemas hápticos. Ainda, é importante identificar a relação de cada pesquisa com a tecnologia háptica, o recurso computacional desenvolvido e as tarefas de geometria. Para cumprir tal proposta, após a introdução, este artigo foi dividido em seis outras seções: a seção 2 trata sobre os fundamentos teóricos da percepção háptica; a seção 3 apresenta as tecnologias hápticas; na quarta seção, o percurso metodológico adotado para a execução desta pesquisa é apresentado; a seção 5 descreve os achados da pesquisa; na seção 6 discutem-se os resultados; e, por fim, a seção 7 apresenta as considerações finais.

\section{Percepção háptica}

O termo "háptico" refere-se à capacidade de uma pessoa sentir, reconhecer e interagir com objetos reais ou virtuais em um ambiente real ou virtual pelo sentido do tato. O sentir e reconhecer as propriedades dos objetos pelo toque estão relacionados às sensações que são convertidas pelo cérebro em informações cutâneas e cinestésicas (Lederman; Klatzky, 2000). Na computação, o háptico é uma tecnologia que permite aplicar as sensações cutâneas e cinestésicas à interação humana com ambientes virtuais. Um dispositivo háptico possibilita às pessoas tocarem em objetos virtuais e sentirem os feedbacks tátil e de força.

Segundo Oakley et al. (2000), as informações cutâneas estão associadas aos sensores da superfície da pele que emitem sensações como: pressão, temperatura, vibração, textura e dor. As cinestésicas estão ligadas aos sensores dos músculos, tendões e juntas que emanam sensações como movimento, dureza, viscosidade, força e forma. A percepção das informações cutâneas e cinestésicas está relacionada com o modo de contato, ou seja, se um objeto é tocado com a mão ou pressionado com o dedo ou explorado na extensão do contorno (Lederman; Klatzky, 2004). Lederman e Klatzky (1987) definiram um conjunto de procedimentos exploratórios (EPs) que é utilizado para explorar um objeto com ou sem visão no intuito de perceber propriedades por meio 
do tato, tais como: textura, rigidez, temperatura, peso, volume, forma global e forma exata. Nesse conjunto de EPs criados por Lederman e Klatzky (1987), foram definidos padrões de movimentos de mãos e dedos caracterizados e relacionados com os conhecimentos sobre o objeto.

Baseando-se nos procedimentos exploratórios de Lederman e Klatzky (1987), a pesquisa de O'Modhrain (1999) teve a intenção de investigar a eficiência da exploração háptica de objetos no mundo real a partir das seguintes limitações: duas mãos, uma mão, dois dedos (polegar e indicador), um dedo (indicador) e uma sonda. Ao realizar a pesquisa sob limitações de exploração, buscou-se mostrar que as tecnologias hápticas utilizadas em ambientes virtuais limitam os usuários no acesso de alguns procedimentos exploratórios que usam uma mão ou duas mãos como modo de contato, pois o efetuador terminal dos dispositivos hápticos é semelhante ao caso mais restrito de exploração: a sonda.

O'Modhrain (1999) ainda busca evidenciar que, ao limitar o acesso de procedimentos exploratórios específicos à percepção de uma determinada propriedade de uma superfície ou objeto, as pessoas podem realizar outros procedimentos alternativos para reconhecê-lo. Nesse sentido, os dispositivos hápticos, abordados na próxima seção, utilizam os procedimentos de Pressure e de Contour de Lederman e Klatzky (1987) para a percepção das propriedades de pressão, temperatura, vibração, textura, movimento, dureza, viscosidade, força e forma.

\section{Tecnologia háptica}

Nesta seção serão apresentados os dispositivos hápticos utilizados em pesquisas científicas na área de interfaces hápticas para o ensino e a aprendizagem de geometria para estudantes cegos, resultante da revisão sistemática.

\subsection{Touch}

Touch é o atual nome do dispositivo háptico Phantom Omni, desenvolvido pela SensAble Technologies em colaboração com a pesquisa realizada no MIT, na década de 1990, pelos pioneiros da indústria Thomas Massie e Dr. Kenneth Salisbury. Atualmente, o dispositivo é comercializado pela empresa 3D Systems. O dispositivo háptico oferece uma navegação tridimensional e feedback de força, oferecendo um sentido de toque em ambientes virtuais com precisão em relação à posição espacial 3D dos eixos $\mathrm{x}, \mathrm{y}$ e $\mathrm{z}$, assim como a orientação do movimento da ferramenta virtual na cena. O Touch possui seis graus de movimento fornecidos por seis pontos do eixo. Todos os graus de movimento têm limites físicos, e quando esses limites forem atingidos, o usuário sentirá uma parada mecânica projetada no dispositivo, conforme mostra a Figura 1, a seguir.

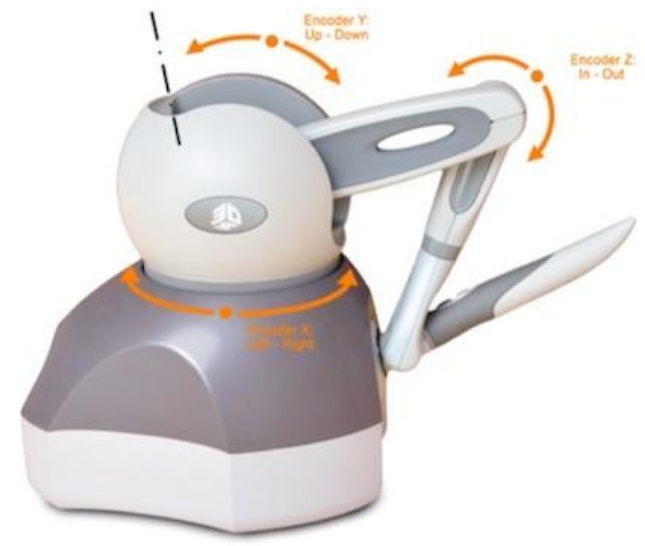

Figura 1 - Seis gruas de movimentos do dispositivo Touch 
O Touch pode ser programado pelo $\mathrm{CHAI3D}$, framework para visualização e simulação de interações hápticas em $\mathrm{C}++$ e multiplataforma. A biblioteca implementa um conjunto de primitivas geométricas $3 \mathrm{D}$ e diversos efeitos hápticos, como magnetismo, superfície, vibração, viscosidade e rigidez.

\subsection{Novint Falcon}

O Novint Falcon é um dispositivo de interação que permite o toque em objetos virtuais de uma cena com três graus de liberdade (uma direção de movimento) e com feedback de força para que os usuários sintam a sensibilidade háptica dos efeitos, das texturas, formas, do peso e das dimensões. Os três graus de liberdade suportados pelo Falcon incluem: movimento direito-esquerdo (X), movimento para cima (Y), movimento para frente e para trás $(\mathrm{Z})$. As direções de movimento do Falcon são conduzidas pelos três motores conectados aos braços articulados, que se prendem a um efetuador terminal no formato de esfera que controla a ferramenta virtual na cena e na colisão com os objetos virtuais, conforme mostra a Figura 2, na sequência.

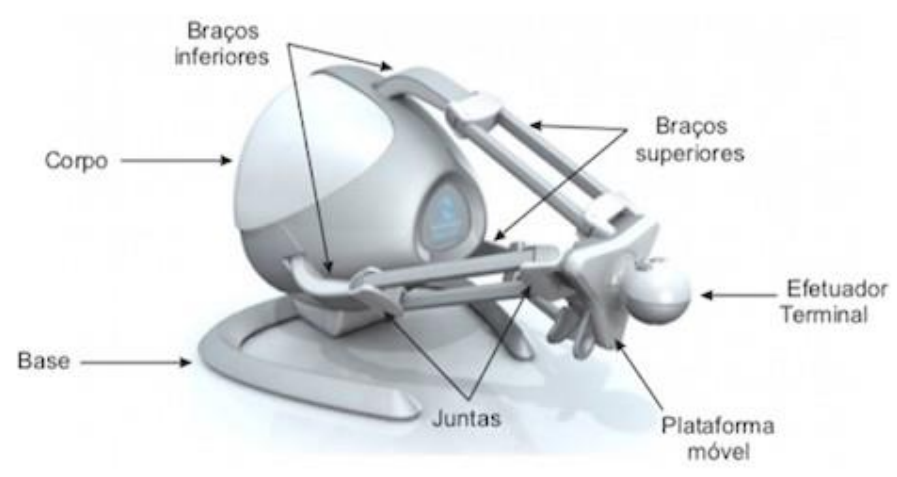

Figura 2 - Membros do dispositivo Novint Falcon

Segundo os pesquisadores da Sandia National Laboratories, quando a ferramenta virtual toca em um objeto no ambiente virtual, o computador registra contato com esse objeto e atualiza as correntes para os motores do dispositivo, criando uma força adequada para que o usuário controle o sensor e sinta o toque 3D de maneira mais realista. De acordo com os pesquisadores do referido laboratório, o Falcon, pequeno robô háptico, dispõe de funções básicas como feedback de força, feedback tátil e propriocepção. $\mathrm{O}$ feedback de força permite ao usuário sentir sensações como peso, forma, movimento e força, por meio dos músculos, tendões e das juntas. O feedback tátil possibilita ao usuário perceber as sensações de pressão, temperatura, vibração, dor e textura pela superfície da pele. Já a propriocepção proporciona ao usuário a percepção da localização espacial do seu corpo sem utilização da visão.

Em um ambiente virtual háptico, os usuários podem controlar a ferramenta virtual e produzir o movimento de localização dos objetos na cena. Fisicamente, o usuário empurra o punho do dispositivo para frente e colide com a ferramenta virtual contra os objetivos. Pelo processo de algoritmos de efeitos hápticos, os motores do Falcon emitem as sensações cutâneas e cinestésicas para dar aos usuários a impressão de toque real.

\section{Percurso metodológico}

O percurso metodológico utilizado para a realização desta revisão sistemática foi guiado pelo processo delineado por Kitchenham (2004), que é composto por três etapas: planejamento da revisão, condução da revisão e documentação dos resultados.

\subsection{Planejamento da revisão}


Com o intuito de nortear a investigação, segundo Connolly et al. (2012), foi concebida a seguinte questão de pesquisa: como sistemas hápticos têm sido empregados para auxiliar usuários cegos no reconhecimento de formas geométricas? Para buscar respostas à questão de pesquisa, foram definidas palavras-chave na língua inglesa em virtude da conformidade com a linguagem das bases de dados escolhidas para a elaboração do protocolo de investigação. Os termos de busca foram definidos como: Haptic Interfaces AND Blind AND recognition AND Geometric shapes.

\subsection{Condução da revisão}

Kitchenham (2004) propõe a condução da revisão nas seguintes etapas: identificação da pesquisa, seleção dos estudos, avaliação da qualidade dos estudos, extração dos dados e síntese dos dados. Na etapa de identificação da pesquisa, a busca foi realizada a fim de atender ao protocolo de investigação entre os anos de 2000 a 2017 nas seguintes bases de dados: Springer, ACM Digital Library (Association for Computing Machinery), Sage Journals e IEEE Xplore Digital Library.

Para conduzir a seleção dos estudos nas bases de dados de acordo com o protocolo de investigação, foi utilizada a ferramenta Mendeley, para apoiar a importação dos trabalhos. Na pré-seleção dos estudos, primeira fase da etapa de avaliação de qualidade, foram aplicados os critérios de inclusão e exclusão na leitura do título e resumo de cada publicação, já na fase de seleção, os critérios foram utilizados na leitura de todo o trabalho. Para isso, foram utilizados um critério de inclusão e dez critérios de exclusão, como demonstra a Tabela 1, a seguir.

Tabela 1 - Critérios de inclusão e exclusão $1^{\mathrm{a}}$ fase

\begin{tabular}{|c|c|}
\hline Critério de inclusão & Critérios de exclusão \\
\hline \multirow{10}{*}{$\begin{array}{l}\text { Sistemas hápticos que } \\
\text { apoiem usuários cegos a } \\
\text { reconhecerem formas } \\
\text { geométricas }\end{array}$} & Sistemas hápticos que não consideram usuários cegos. \\
\hline & Percepção tátil por usuários cegos por meio de protótipos físicos. \\
\hline & $\begin{array}{l}\text { Sistemas computacionais que consideram a interface de toque } \\
\text { (touch screen) por dispositivos móveis ou gestos para usuários } \\
\text { cegos. }\end{array}$ \\
\hline & $\begin{array}{l}\text { Design de interfaces acessíveis para cegos em sistemas } \\
\text { computacionais. }\end{array}$ \\
\hline & $\begin{array}{l}\text { Interfaces de sistemas que abordam técnicas de atuadores para } \\
\text { movimento. }\end{array}$ \\
\hline & $\begin{array}{l}\text { Sistemas computacionais que consideram o GPS para detecção e } \\
\text { reconhecimento de obstáculos e objetos em movimento por } \\
\text { usuários cegos. }\end{array}$ \\
\hline & Protótipo/desenvolvimento de dispositivos hápticos. \\
\hline & Percepção háptica humana. \\
\hline & $\begin{array}{l}\text { Interfaces de sistemas que não abordam percepção háptica por } \\
\text { meio de dispositivo. }\end{array}$ \\
\hline & Levantamento de requisitos para sistemas hápticos. \\
\hline
\end{tabular}
Fonte: Autoria própria.

$\mathrm{Na}$ etapa de avaliação de qualidade dos estudos, a fase de pré-seleção utilizou os critérios de inclusão e exclusão supracitados, tendo sido selecionados 25 estudos, conforme mostra a Tabela 2, na sequência.

Tabela 2 - Resumo da seleção e avaliação da qualidade dos estudos

\begin{tabular}{ccccccc}
\hline $\begin{array}{c}\text { Base de } \\
\text { dados }\end{array}$ & $\begin{array}{c}\text { Resultado } \\
\text { inicial }\end{array}$ & Duplicados & Fase de pré-seleção & Fase de seleção \\
\hline Springer & 42 & 0 & Inclusão & Exclusão & Inclusão & Exclusão \\
\hline
\end{tabular}




\begin{tabular}{|c|c|c|c|c|c|c|}
\hline \multirow[t]{2}{*}{$\begin{array}{c}\text { Base de } \\
\text { dados }\end{array}$} & \multirow[t]{2}{*}{$\begin{array}{c}\text { Resultado } \\
\text { inicial }\end{array}$} & \multirow[t]{2}{*}{ Duplicados } & \multicolumn{2}{|c|}{ Fase de pré-seleção } & \multicolumn{2}{|c|}{ Fase de seleção } \\
\hline & & & Inclusão & Exclusão & Inclusão & Exclusão \\
\hline $\begin{array}{l}\text { ACM Digital } \\
\text { Library }\end{array}$ & 7 & 0 & 7 & 0 & 1 & 5 \\
\hline Sage Journals & 5 & 0 & 1 & 4 & 0 & 1 \\
\hline $\begin{array}{l}\text { IEEE Xplore } \\
\text { Digital Library }\end{array}$ & 7 & 0 & 5 & 3 & 2 & 2 \\
\hline Total & 61 & 0 & 25 & 37 & 7 & 15 \\
\hline
\end{tabular}

Na fase de seleção, conforme demonstrado na Tabela 2, restaram sete trabalhos em virtude da utilização de novos critérios de inclusão e exclusão, mostrados na Tabela 3 , a seguir, que refinaram as escolhas do pesquisador após a leitura completa dos trabalhos.

Tabela 3 - Critérios de inclusão e exclusão após a leitura completa dos trabalhos

\begin{tabular}{|l|l|}
\hline Critério de inclusão & \multicolumn{1}{c|}{ Critérios de ex clu são } \\
\hline \multirow{5}{*}{$\begin{array}{l}\text { Sistemas hápticos que } \\
\text { apoiem usuários cegos a } \\
\text { reconhecerem formas } \\
\text { geométricas 2D ou 3D. }\end{array}$} & $\begin{array}{l}\text { Mapas táteis em ambientes virtuais hápticos. } \\
\text { usuários cegos. }\end{array}$ \\
\cline { 2 - 2 } & $\begin{array}{l}\text { Sistemas hápticos que abordam a exploração de modelos virtuais } \\
\text { 3D sem considerar o reconhecimento da forma do objeto. }\end{array}$ \\
\cline { 2 - 2 } & $\begin{array}{l}\text { Sistemas hápticos que abordam a percepção de textura de objetos. } \\
\text { componentes de planilhas eletrônicas. }\end{array}$ \\
\cline { 2 - 2 } & $\begin{array}{l}\text { Sistemas hápticos que permitem fazer e reconhecer desenhos e } \\
\text { imagens. }\end{array}$ \\
\cline { 2 - 2 } & $\begin{array}{l}\text { Orientação e mobilidade em mundos virtuais hápticos sem o } \\
\text { reconhecimento das formas dos objetos. }\end{array}$ \\
\cline { 2 - 2 } & Sistemas hápticos para compreender gestos. \\
\cline { 2 - 2 } & Sistemas hápticos (luvas) para explorar páginas web pelo toque. \\
\cline { 2 - 2 } & $\begin{array}{l}\text { Sistemas hápticos que abordam a exploração dos elementos da } \\
\text { interface. }\end{array}$ \\
\hline
\end{tabular}

Fonte: Autoria própria.

\subsection{Documentação do resultados}

Cada trabalho selecionado foi analisado com a finalidade de identificar os seguintes elementos: área de aplicação, tecnologia háptica, recurso didático computacional desenvolvido, tarefa de geometria e resultados, conforme mostra a seção 5, na sequência, a fim de explorar as contribuições e as lacunas para trabalhos futuros. Dentre os sete trabalhos encontrados na revisão sistemática, aqui serão apresentadas quatro pesquisas que estão relacionadas com a área de ensino e aprendizagem de geometria.

\section{Resultados}

Gouy-Pailler et al. (2007) no trabalho intitulado A Haptic Based Interface to Ease Visually Impaired Pupils' Inclusion in Geometry Lessons, desenvolveram um editor multimodal integrado ao dispositivo Phantom Omni para o ensino de geometria plana com as tarefas de construção e percepção de formas geométricas. Após o primeiro experimento, todos os participantes da pesquisa conseguiram reproduzir os gestos propostos, embora alguns fossem mais difíceis de reproduzir. Já os resultados do 
segundo experimento corroboram a ideia dos pesquisadores, que propõem o uso de uma interface baseada em caneta háptica como apoio aos alunos com deficiência visual para construírem formas geométricas usando gestos de comandos.

Shimomura, Hvannberg e Hafsteinsson (2013), no estudo intitulado Haptic Cues as a Utility to Perceive and Recognise Geometry, desenvolveram um ambiente virtual integrado ao dispositivo Phantom Omni para o ensino de geometria espacial com as tarefas de conceitualizar as propriedades da esfera, do cubo, do cilindro, do cone, dos toros e do cubo girando. Demonstraram que, após dois experimentos, os usuários podem facilmente reconhecer e manipular com recursos hápticos objetos familiares, embora com alguma ajuda.

Stamm, Altinsoy e Merchel (2011), no estudo intitulado Influence of the Auditory Localization Direction on the Haptic Estimation of Virtual Length, produziram um ambiente virtual com cubos integrado ao dispositivo Phantom Omni para o ensino de geometria espacial com as tarefas de identificação de características dos cubos. Após o experimento, os participantes da pesquisa conseguiram perceber o comprimento dos cubos pelo uso do dispositivo háptico.

Uma abordagem distinta foi proposta por Petridou, Blanchfield e Brailsford (2011) na pesquisa intitulada Involving the User with Low or no Vision in the Design of an Audio-haptic Learning Environment for Learning about 3D Shapes: The First Approach. Os autores criaram um jogo educacional com feedback de força integrado ao dispositivo Novint Falcon para o ensino de geometria espacial com as tarefas de localizar e nomear o objeto e perceber as texturas. Durante o trabalho, foi realizado um experimento na busca de respostas para localizar e nomear o objeto e saber qual textura fornece sugestões táteis mais exatas. Os resultados mostraram que os estudantes precisavam de um objeto com textura mais distinta para estarem cientes da posição de sua mão virtual. As texturas magnéticas e rochosas eram as favoritas, com a última produzindo pistas mais precisas e claras, e a primeira mantendo o usuário constantemente no objeto.

\section{Discussão}

Os ambientes virtuais com uso da tecnologia háptica podem proporcionar espaços de ensino e aprendizagem para o desenvolvimento das habilidades relacionadas às formas geométricas nos estudantes cegos, com base em vários cenários de problemas, contextualizados com o mundo real ou com a própria ciência matemática.

Segundo a visão de articular método de ensino e ferramentas adequadas, como referido na Base Nacional Comum Curricular (BNCC), os resultados obtidos em relação ao ensino e à aprendizagem de estudantes cegos em geometria com o uso de tecnologias hápticas apresentados nos trabalhos de Petridou, Blanchfield e Brailsford (2011), Stamm, Altinsoy e Merchel (2011) e Shimomura, Hvannberg e Hafsteinsson (2013) mostraram-se relevantes para o desenvolvimento dos pensamentos geométrico e computacional. Além dos benefícios para o ensino e a aprendizagem, o uso da tecnologia háptica pode promover a colaboração e o trabalho em equipe, uma vez que os estudantes com ou sem visão terão um ponto de partida comum de comunicação e compreensão.

$\mathrm{Na}$ pesquisa de Petridou, Blanchfield e Brailsford (2011), o ambiente oportunizou aos estudantes cegos a prática de interagir com formas geométricas tridimensionais para investigar sua localização, seu nome e suas texturas com a intenção de desenvolver o raciocínio espacial e a compreensão compartilhada do material didático.

Já no trabalho de Stamm, Altinsoy e Merchel (2011), o ambiente possibilitou aos estudantes cegos, pelo sentido do tato, o reconhecimento do comprimento dos cubos, e pelo sentido auditivo, a pista de localização para o início do movimento de exploração. $\mathrm{Na}$ investigação de Shimomura, Hvannberg e Hafsteinsson (2013), pode-se observar que o ambiente virtual permitiu aos estudantes cegos a identificação de propriedades das formas com auxílio de efeitos hápticos. 
Por fim, as tecnologias hápticas de hardware e software, apresentadas nas pesquisas, permitem a compreensão do material didático pelos estudantes cegos, no que tange ao estudo das formas geométricas. Essa compreensão permite ao professor a construção de habilidades específicas da geometria plana e espacial de forma a desenvolver a competência do pensamento geométrico.

Em termos de limitações, observou-se que o ambiente virtual háptico proposto por Gouy-Pailler et al. (2007) tem suas contribuições quanto ao uso da tecnologia háptica no ensino e na aprendizagem de alguns conceitos de geometria plana, mas deixa lacunas em relação ao estudo da geometria espacial.

O trabalho de Shimomura, Hvannberg e Hafsteinsson (2013) é, talvez, o que traz maior contribuição para a área da geometria no Ensino Fundamental em relação ao estudo das formas, no entanto distancia-se da abordagem proposta pela BNCC no que se refere aos conteúdos e habilidades, pois o ambiente virtual háptico busca auxiliar os estudantes cegos em parte dos conteúdos de geometria espacial, como: definir as propriedades da esfera, do cubo, do cilindro, do cone, dos toros e do cubo girando. No contexto brasileiro, tais considerações podem ser importantes no que diz respeito ao emprego dos dispositivos hápticos no apoio à aprendizagem de geometria no ensino fundamental.

Já o trabalho criado por Petridou, Blanchfield e Brailsford (2011), de modo similar à pesquisa de Shimomura, Hvannberg e Hafsteinsson (2013), traz contribuições quanto ao uso do dispositivo háptico no estudo das formas, mas deixa lacunas na completude dos conteúdos que devem ser abordados, pois o ambiente virtual busca apoiar os estudantes cegos nos seguintes conteúdos: identificar os nomes das formas geométricas espaciais e identificar nos sólidos geométricos figuras geométricas planas.

\section{Considerações finais}

A principal contribuição desta revisão sistemática de literatura foi demonstrar como as tecnologias hápticas de hardware e software, apesar das limitações de exploração, como destacou O'Modhrain (1999), permitem a interação dos estudantes cegos com os objetos virtuais por meio dos procedimentos de Pressure e de Contour de Lederman e Klatzky (1987) possibilitados pelos dispositivos hápticos e a percepção das características das formas geométricas planas e espaciais pelos feedback tátil e de força.

Grande parte dos trabalhos analisados demonstrou que a tecnologia háptica pode proporcionar espaços de ensino e aprendizagem que auxiliam o estudante cego no processo de aprendizagem de geometria. Contudo, constatou-se que os trabalhos selecionados pela revisão sistemática deixam lacunas em relação aos conteúdos e abordagens propostas pela BNCC ao ensino e aprendizagem de geometria para o Ensino Fundamental $\left(2^{\circ}\right.$ ano), possibilitando assim novas pesquisas na área da Informática na Educação que contemplem os conteúdos do currículo e as tecnologias hápticas de hardware e software como forma de oportunizar a compreensão dos conceitos de geometria pelos estudantes cegos.

\section{Referências Bibliográficas}

ARGYROPOULOS, V. S. Tactual shape perception in relation to the understanding of geometrical concepts by blind students. The British Journal of Visual Impairment, Londres, p. 7-16, 2002. Disponível em: <https://journals.sagepub.com/doi/abs/10.1177/026461960202000103?journalCode=jvi b>. Acesso em: 10 out. 2018 .

FERRONATO, R. A construção de instrumento de inclusão no ensino da Matemática. Florianópolis: U̧FSC, 2002. Dissertação de Mestrado.

GOUY-PAILLER, C.; ZIIP-ROUZIER, S.; VIDAL, S.; CHÊNE, D. A Haptic Based Interface to Ease Visually Impaired Pupils' Inclusion in Geometry Lessons. In: 
STEPHANIDIS, C. (Ed.) Universal Access in Human-Computer Interaction. Applications and Services. UAHCI 2007. Lecture Notes in Computer Science. Berlin: Heidelberg, 2007. v. 4556. Disponível em:

<https://link.springer.com/10.1007/978-3-540-73283-9_66>. Acesso em: 22 nov. 2017.

KITCHENHAM, B. Procedures for performing systematic reviews. Technical Report Technical Report TR/SE-0401. Keele University and NICTA, 2004.

LEDERMAN, S. J.; KLATZKY, R. L. Haptic identification of common objects: Effects of constraining the manual exploration process. Perception \& Psychophysics, v. 66, Issue 4, p. 618-628, 2004. Disponível em: <https://link.springer.com/article/10.3758/BF03194906>. Acesso em: 22 out. 2018.

; Modality specificity in cognition: The case of touch. The Nature of Remembering: Essays in Honor of Robert G. Crowder. Washington: American Psychological Association Press, 2000.

Hand movements: A window into haptic object recognition. Cognitive Psychology, v. 19, p. 342-368, 1987. Disponível em: <https://www.sciencedirect.com/science/article/pii/0010028587900089>. Acesso em: 8 out. 2018 .

LIRIO, S. B. A tecnologia informática como auxílio de geometria para deficientes visuais. Rio Claro: Universidade Estadual Paulista, 2006. 115 f. Dissertação de Mestrado.

O'MODHRAIN, M. S. Restricted Access: Exploratory Procedures and Object Properties. Proc. ASME International Mechanical Engineering Congress: Dynamic Systems and Control Division. v. 2. Haptic Interfaces for Virtual Environments and Teleoperator Systems. DSC- v. 61, 1999. Disponível em: <http://ccrmawww.stanford.edu/ sile/abstracts/asme99.html>. Acesso em: 23 nov. 2018.

OAKLEY, I.; MCGEE, M.; BREWSTER, S. A.; GRAY, P. D. Putting the feel in look and feel. CHI '00 Proceedings of the SIGCHI conference on Human Factors in Computing Systems, New York, p. 415-422, 2000.

Doi: $10.1145 / 332040.332467$.

PETRIDOU, M.; BLANCHFIELD, P.; BRAILSFORD, T. Involving the user with low or no vision in the design of an audio-haptic learning environment for learning about 3D shapes: The first approach. In: COMPUTER SCIENCE AND ELECTRONIC ENGINEERING CONFERENCE (CEEC), 3, 2011, Colchester. Annals.

Colchester, 2011. Disponível em: <https://ieeexplore.ieee.org/document/5995820>. Acesso em: 28 set. 2017.

SHIMOMURA, Y.; HVANNBERG, E. T.; HAFSTEINSSON, H. Haptic cues as a utility to perceive and recognize geometry. Universal Access in the Information Society, v. 12, Issue 2, p 125-142, 2013. Disponível em:

<https://link.springer.com/10.1007/s10209-012-0271-2>. Acesso em: 28 set. 2017.

STAMM, M.; ALTINSOY, M. E.; MERCHEL, S. Influence of the Auditory Localization Direction on the Haptic Estimation of Virtual Length. In: COOPER, E. W.; KRYSSANOV, V. V.; OGAWA, H.; BREWSTER, S. (Ed.). Haptic and Audio Interaction Design. HAID 2011. Lecture Notes in Computer Science. Berlin: Heidelberg, 2011. v. 6851. Disponível em: <https://link.springer.com/10.1007/978-3-642-22950-3_11>. Acesso em: 22 nov. 2017. 